\title{
Dynamic generation of personalized hybrid recommender systems
}

\author{
Simon Dooms
}

Supervisor(s): Luc Martens

\section{INTRODUCTION}

With the rising overabundance of information people are experiencing difficulties in selecting relevant content. Recommender systems try to assist users in this process by using intelligent recommendation algorithms to filter the information.

Nowadays recommender systems focus on combining multiple recommendation algorithms to smooth out disadvantages and to exploit their benefits [1]. Although these hybrid systems have shown their value [2], they often suffer from overfitting and do not allow individual user oriented hybrid approaches.

We would like to come up with a new recommendation strategy that allows hybrid recommendation approaches to be dynamically generated for individual users.

\section{Methods}

Through an online experiment we will first try to evaluate the value of different types of feedback systems and consider an optimal feedback collection strategy for hybrid recommender systems. Next we will use the collected dataset from this experiment to analyze the applicability of machine learning techniques for the specific optimization problem we need to overcome in this research.

S. Dooms is a member of the Wireless and Cable group of the Department of Information Technology, Ghent University (UGent), Gent, Belgium. E-mail: simon.dooms@intec.ugent.be.

\section{ONLINE FEEDBACK EXPERIMENT}

We have set up an online feedback experiment on a popular Belgian cultural website that offers an overview of upcoming events in Flanders (the northern part of Belgium).

In May 2010 we installed a module that logs all kinds of possible user feedback information. Explicit feedback is collected by four rating systems that are randomly shown to browsing users. Browsing behavior and the clicks on specific links are some of the forms of implicit feedback we monitor. In five months time we already registered over 8000 ratings, 800.000 pageviews and more than 200.000 unique clicks.

\section{Conclusions}

The collected data will allow us to analyze the value and optimal feedback collection approaches for hybrid recommender systems. The results can be integrated in a valuable dataset for the planned research regarding machine learning techniques to personalize the hybrid recommendation process.

\section{REFERENCES}

[1] Porcel, C., Lpez-Herrera, A. G., and HerreraViedma, E. 2009. A recommender system for research resources based on fuzzy linguistic modeling. Expert Syst. Appl. 36, 3 (Apr. 2009), 51735183 .

[2] Jahrer, M., Tscher, A., and Legenstein, R. 2010. Combining predictions for accurate recommender systems. In Proceedings of the 16th ACM SIGKDD international Conference on Knowledge Discovery and Data Mining (Washington, DC, USA, July 25 - 28, 2010). KDD '10. ACM, New York, NY, 693-702. 